\title{
Investigating the Antioxidant Capacity of Fruits and Fruit Byproducts through an Introductory Food Chemistry Experiment for High School
}

\author{
Cristina Soares \\ Manuela Correia \\ Cristina Delerue-Matos and \\ M. Fátima Barroso
}

\section{Abstract}

This paper reports a laboratorial internship included in the Portuguese Science and Technology promotion program "Internships for Young People in Laboratories (Ciência Viva no Laboratório)", which provided high school students an opportunity to approach the reality of scientific and technological research in a higher education institution. During this internship, students acquired knowledge related to the assessment of the total antioxidant capacity (TAC) of fruits and their byproducts while learning techniques, such as molecular spectrophotometry, as an analytical methodology to measure TAC using the ferric reducing antioxidant power (FRAP) and the total phenolic content (TPC) assays. First, the students were introduced to solid-liquid extraction as a conventional technique to extract antioxidants from the selected matrices. In order to optimize the extraction yield, different solvents, temperatures, and extraction times were used. Then, the students developed skills on TPC and FRAP assays by performing calibration curves using standard antioxidants, namely, gallic acid and ascorbic acid, prior to the measurement of the TAC of fruits (apple and orange) and respective byproducts. Final analysis included TAC values for comparison between fruits and their byproducts and also the influence of the extraction conditions on the TAC levels. At the end, the students presented their findings in a scientific poster and in a postlaboratory quiz. This laboratorial internship has been carried out since 2012 and was designed to be performed during a week, $7 \mathrm{~h}$ per day, in groups of two young students.

\section{Keywords:}

Analytical Chemistry; Hands-On Learning/Manipulatives; High School/Introductory Chemistry; Instrumental Methods; Laboratory Instruction; Natural Products; Oxidation/Reduction; Quantitative 


\section{Introduction}

According to Laguerre and collaborators, "a biological antioxidant is defined as a substance that, when present at low concentrations compared to an oxidizable substrate, protects, by itself and through its oxidation products, that substrate from oxidation, and ultimately protects the organism from harmful effects of oxidative stress".(1) Actually, oxidative stress, arising as a result of an imbalance between free radical (namely, reactive oxygen species, ROS) production during the metabolic and inflammatory living process, and antioxidant defenses, is associated with damage to a wide range of molecular species including lipids, proteins, and nucleic acids which is frequently associated with many complex pathologies (cardiovascular diseases, inflammatory disorders, and cancer).(2, 3)

Certain diseases, cancer in particular, are less common among people who eat plenty of fruit and vegetables, and it has been suggested that the health benefits are due to the antioxidants they contain which cancel out the harmful effects of ROS.(4) Fruits and vegetables contain a variety of antioxidants that include phenolic compounds,(5) vitamins C and E, carotenoids, chlorophylls, and flavonoids.(6) The antioxidants present in fruits and vegetables will vary from species to species and within cultivars but also from some external factors ranging from how the plant is grown and harvested to how it is packaged and stored in the market.(7)

Fruits, namely, citrus and apples, are consumed in high quantities all over the world in natural and peeled forms and as juice. In the case of juice production, after its extraction from citrus and apple, there remains a byproduct (around $50 \%$ and $35 \%$ of the fresh citrus and apple weight, respectively) consisting of peel, pulp, rag, and seeds. The disposal of the byproducts is becoming a major problem for many factories which consider that fruit residues do not have economic value. However, it has been reported that fruit byproducts potentially represent a rich source of natural flavonoids, and phenolic compounds. $(8,9)$

For assessment of the quantity and class of antioxidants present in fruits and vegetables, several methods have been used in recent years that involve the evaluation of the total antioxidant capacity (TAC) and/or the individual identification and quantification of antioxidants. Some of the most used methods are in vivo, in vitro, chemiluminescence, chromatography, $(10,11)$ electrochemical methods,(12) infrared spectroscopy, UV-vis spectroscopy,(13, 14) and mass spectrometry, among others. However, before quantification, a very important step is the extraction of the antioxidant compounds from the food matrix. The most used extraction techniques are the use of organic solvents, supercritical fluids, microwave extraction, subcritical water extraction, or ultrasonic extraction.(15)

\section{Students Learning Goals}


The chemistry and physics curriculum for high school students in Portugal includes spectroscopy, electronic transitions, photochemical reactions, free radical formation and stability, antioxidants, and the electromagnetic spectrum in the 10th grade. This work, "Fruits byproducts: are they valuable?", has been proposed, since 2012, for 10th and 11th grade students in the framework of the program "Internships for Young People in Laboratories (Ciência Viva no Laboratório)(16)—Science in the Holidays for Young People" that provides high school students an opportunity to approach the reality of scientific and technological research while joining research teams in higher education institutions. Ongoing since 1997, this initiative has involved more than 11,000 students who attended traineeships in scientific and technological institutions across the country, in all scientific areas.(17)

Regarding the traineeship discussed in this paper, in a weeklong, $7 \mathrm{~h}$ per day training, students are introduced to molecular spectrophotometry as conventional methodology for the assessment of the TAC of fruits and their byproducts by using the ferric reducing antioxidant power (FRAP) and the total phenolic content (TPC) methodologies.(18-20) The extraction of the antioxidants from the selected matrices was performed using the solid-liquid extraction technique with different solvents, temperatures, and extraction times.

As a result of performing these experiments, it is expected that students:

$\checkmark$ Increase their knowledge concerning antioxidants and their importance to counteract and prevent the adverse effects of ROS;

$\checkmark$ Demonstrate that the analyzed fruits and fruit byproducts are rich in antioxidants;

$\checkmark$ Carry out effective laboratorial experiments to analyze foodstuffs;

$\checkmark$ Understand the basic principles of molecular spectroscopy and the use of more advanced instrumentation like multiplate readers;

$\checkmark$ Identify the chemical principles used on the protocols for the FRAP and TPC determinations;

$\checkmark$ Statistically analyze FRAP and TPC results, and

$\checkmark$ Develop the ability to present their findings in a scientific poster format.

\section{Experimental Section}

\section{Extraction of Antioxidants from Fruit Samples}


During a week, 4-6 students were divided in groups of two, and first, the students analyzed and read the student manual "Fruits by-products: are they valuable?" (available in the Supporting Information). Finally, they were introduced to an assessment of the antioxidant properties of selected fruits and their byproducts. The chosen fruits were fresh oranges (analyzed as the fruit pulp and the peel) and fresh apples analyzed as whole fruit (pulp and peel). Fruits were cut into small pieces and ground in an electric grinder. The water content of each sample was determined in a moisture balance. For antioxidant extraction, $1.5 \mathrm{~g}$ of fruit material was placed into a Falcon tube, and $20 \mathrm{~mL}$ of solvent were added and the mixture homogenized. Different solvents, temperatures, and extraction times were used, according to the Student Manual presented in the Supporting Information (Student Manual S1). The supernatant was filtered and transferred to a new tube and the tube wrapped in aluminum foil and stored at $4{ }^{\circ} \mathrm{C}$ until analysis.

\section{Determination of Total Phenolic Content (TPC) and FRAP Assay}

The TPC of the fruit and respective byproduct extracts was determined by the Folin-Ciocalteu assay, and the TAC was measured using the FRAP protocol. A complete description of the used methodologies is presented in the Supporting Information (Student Manual S1).

\section{Hazards}

The Folin-Ciocalteu (FC) reagent is a commercial mixture of sodium tungstate, sodium molybdate, phosphoric acid, hydrochloric acid, lithium sulfate, and water. It is corrosive and dangerous if inhaled or if it is in contact with any part of the body. All wastes generated from the FC test should be disposed of in accordance with best laboratory practices. Aqueous ethanol is flammable. Gallic acid is hazardous in contact with the eyes and after ingestion. 2,4,6-Tripyridyl-s-triazine (TPTZ) can cause skin, eye, and respiratory irritation and allergic reaction.

$\mathrm{HCl}$ and $\mathrm{CH} 3 \mathrm{COOH}$ cause severe skin burns and eye damage, and may cause respiratory irritation. Some precaution should be taken such as avoiding breathing vapors, wearing protective gloves and protective clothing, and protecting the eyes and face. To prepare the $\mathrm{HCl}$ or $\mathrm{CH} 3 \mathrm{COOH}$ solutions, fill the volumetric flask about halfway with deionized water to avoid violent reactions. Never add water to concentrated acid.

\section{Results and Discussion}

The results presented and discussed in this section were obtained for the three groups of students that attended the traineeship in 2015. In the initial module, students performed the sample description and the sample preparation (the samples were grinded and homogenized), and the measurement of the water content of the samples using an infrared moisture balance. Students observed that the orange pulp, orange peel, and apple (pulp and peel) presented an average 
experimental moisture content of $89 \%, 74 \%$, and $79 \%$, respectively. These results were used to calculate the TPC and FRAP values per gram of dry sample.

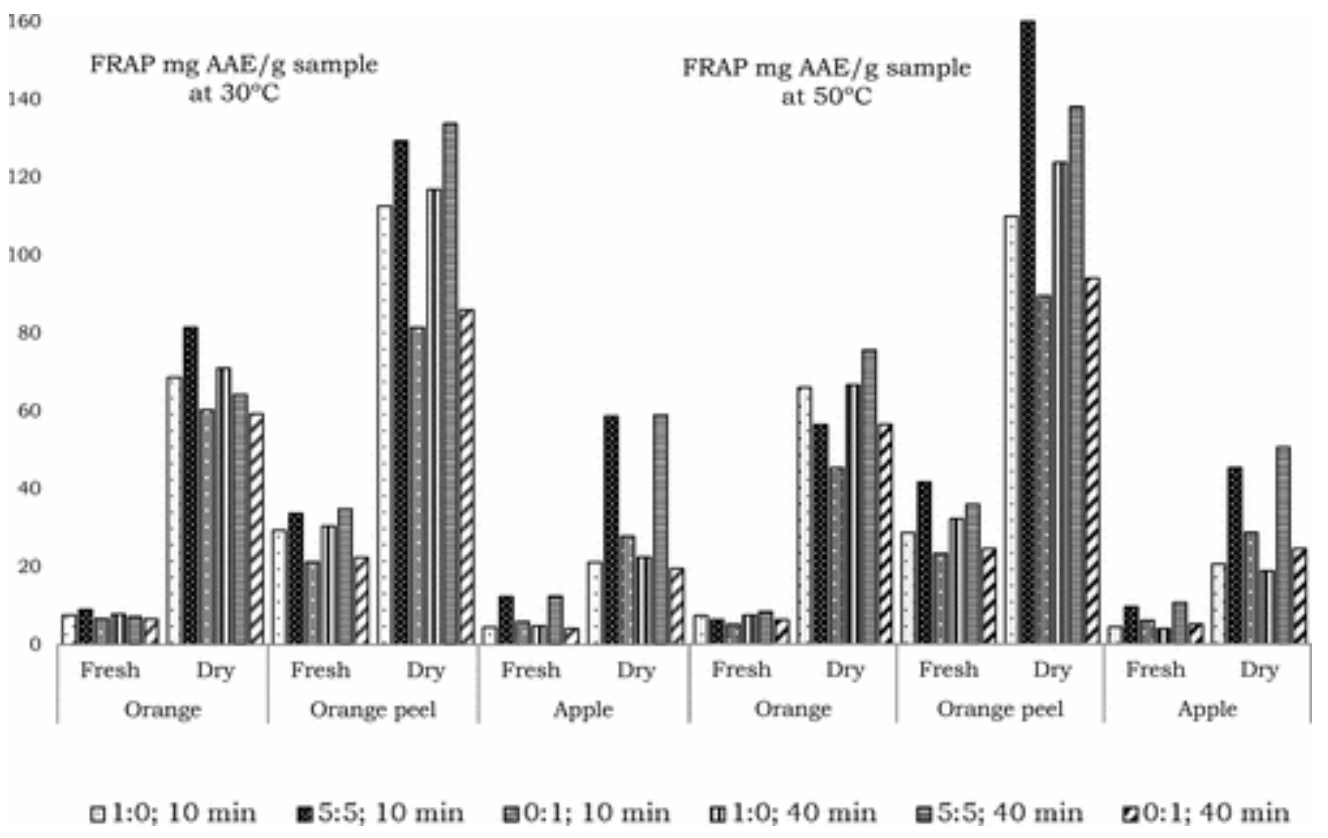

Figure 1. TPC values for fruit samples in $\mathrm{mg}$ GAE/g of fresh or dry sample. The extraction conditions with different water/ethanol ratios (1:0; $5: 5 ; 0: 1)$ for 10 or 40 min at 30 and $50{ }^{\circ} \mathrm{C}$ are presented and compared.

Then, the students used different extraction conditions (Student Manual S1) to analyze and compare the extraction efficiencies and their effect in the FRAP and TPC levels. The results obtained by students are shown in Figures 1 and 2. Students expressed the TPC values in terms of gallic acid equivalents (GAE)/gsample and the FRAP results as ascorbic acid equivalents (AAE)/gsample for apple (whole fruits), orange pulp, and orange peels (Tables S1-3, respectively, in the Supporting Information). Students analyzed the obtained results, and they concluded that, for the extraction temperatures (30 or $50{ }^{\circ} \mathrm{C}$ ) and times (10 or $40 \mathrm{~min}$ ) tested, the best extraction solvent was, in general, the mixture of water/ethanol (50:50, v/v). Moreover, TPC increased, when the extraction time and the temperature also increased. Similar results were obtained by Rusak et al.; they extracted polyphenols from tea leaves using hot water and mixtures of water/ethanol at different extraction times.(20) 


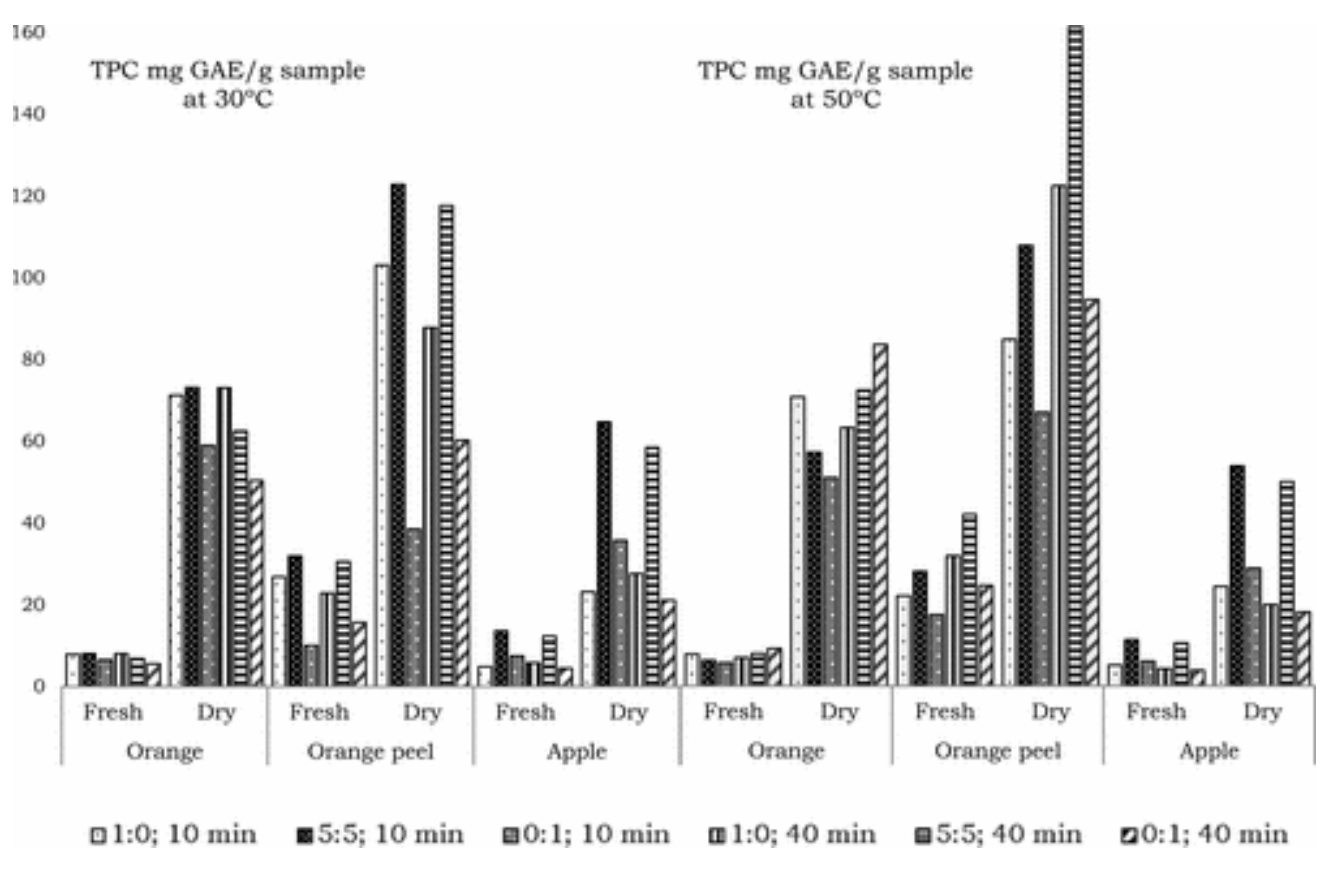

Figure 2. FRAP values for fruit samples in $\mathrm{mg} A A E / g$ of fresh or dry sample. The extraction conditions with different water/ethanol ratios (1:0; 5:5; 0:1) for 10 or 40 min at 30 and $50^{\circ} \mathrm{C}$ are presented and compared

Whenever possible, the students were encouraged to compare their findings with those reported in the scientific literature, and they have verified that this result is in accordance with that reported in other works.(15)

Fruits samples present, in general, high amounts of polyphenols and high TAC.(15) In this work, apple samples presented similar TPC (3.8-12.3 mg GAE/g of fresh sample) (Figure 1) and similar values for TAC measured by the FRAP method (3.9-12.4 mg AAE/g of fresh sample) (Figure 2). Students compared these results with those of Valavadinis et al.(21) who analyzed apples (whole fruit and peels) comparing 5 cultivars from organic and conventional agricultural practices using $90 \%$ methanol as the extraction solvent, at room temperature. Valavadinis and collaborators reported for TPC, for the whole fruits, values that ranged between 0.80 to $1.96 \mathrm{mg} \mathrm{GAE} / \mathrm{g}$ of fresh sample. Using the FRAP assay, the same authors reported TAC values for the same apple varieties and for the whole fruits between 0.37 and $1.57 \mathrm{mg} \mathrm{AAE} / \mathrm{g}$ of fresh sample.

Regarding orange samples, TPC values obtained by students were between 5.5 and $9.2 \mathrm{mg}$ GAE/g fresh sample (50.4-83.7 mg GAE/g dry sample), and FRAP values ranged between 4.9 and $8.4 \mathrm{mg}$ AAE/g of fresh sample (44.4-76.4 mg AAE/g of dry sample) for orange pulp.

Orange peels presented the highest values for TPC and for FRAP: TPC, 10.0-42.0 mg GAE/g fresh sample (38.5-161.5 mg GAE/g dry sample); and FRAP, 20.1-40.8 mg AAE/g fresh sample (77.2$157.1 \mathrm{mg} \mathrm{AAE} / \mathrm{g}$ dry sample). 
In their experiments, the students attending this traineeship could conclude that the extraction solvent composition, and extraction time and temperature, affects analyte extraction from the matrices. Therefore, when comparing their own results with literature values, they were asked to check the experimental conditions of extraction, the standard antioxidant used in the construction of the calibration curves, and the units in which results were presented (e.g., dry or fresh weight).

Students analyzed the work performed by Goulas et al.(22) which used boiling ethyl acetate to extract antioxidants from different citrus fruits and residues and reported for TPC $108.5 \mathrm{mg}$ GAE/g of dry sample for the orange pulp and $196.2 \mathrm{mg} \mathrm{GAE} / \mathrm{g}$ dry sample for the orange peel. For FRAP, the same authors reported $51 \mathrm{mg} \mathrm{AAE} / \mathrm{g}$ dry sample for the pulp and $79 \mathrm{mg} \mathrm{AAE} / \mathrm{g}$ dry sample.

Students found it difficult to compare their results with other reports because the extraction of polyphenolic compounds was not performed under the same conditions, namely, the extraction solvent which can significantly affect the set of compounds that is extracted from the matrix. Additionally, within the same fruit species, there may be antioxidant diversity and different susceptibility to oxidation and hydrolysis. Other factors that can influence quantification are the fruit variety, environmental conditions, the degree of fruit ripening, and genetic factors, as well as many parameters related to the extraction method (temperature, contact time, solvent to solid ratio, solvent type, etc.).(19)
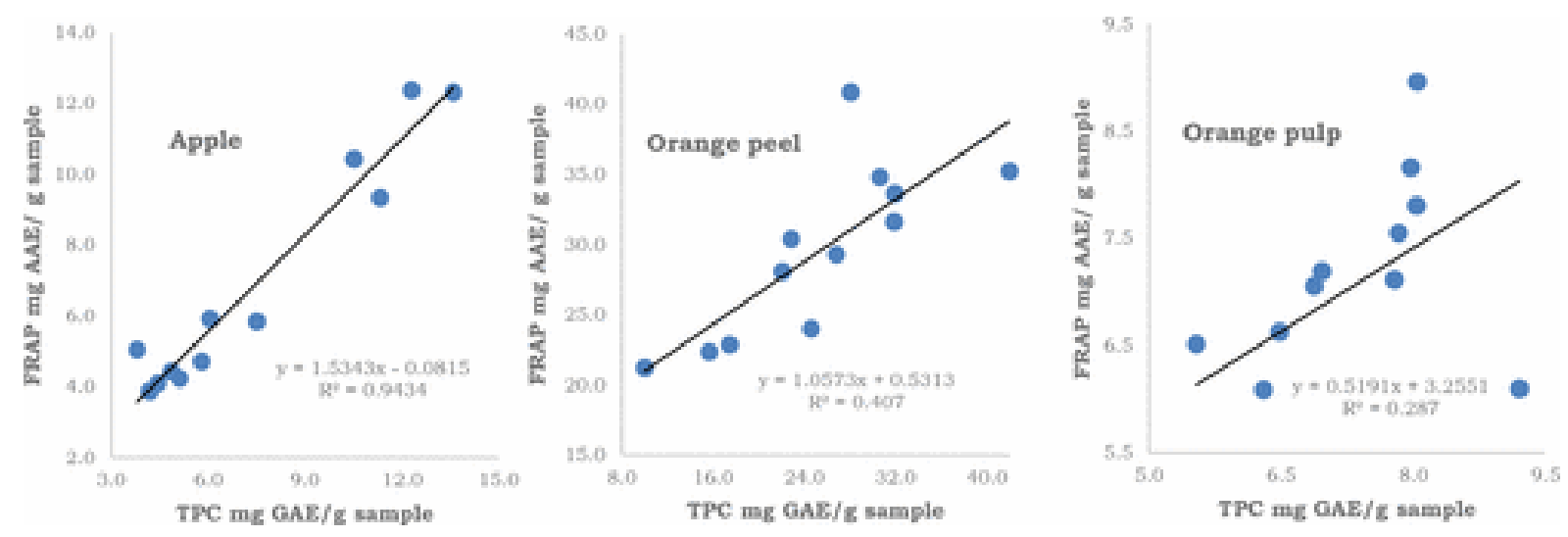

Figure 3. Correlation analysis between TPC (mg GAE/g fresh sample) and FRAP ( $m g$ AAE/g fresh sample) results for apples, orange peel, and orange pulp.

Students carried out a correlation between TPC and TAC values (Figure 3), and poor or lack of correlation ( $r 2=0.407$ and 0.287 , respectively) was observed for orange peel and orange pulp, similar to other reports.(19) According to Goulas et al.,(22) this weak correlation can be attributed to the fact that some nonphenolic compounds may contribute to the overall antioxidant activity of the extracts, which are not accounted for by the Folin-Ciocalteu assay. In addition, the Folin-Ciocalteu reagent may react with interfering substances like ascorbic acid, citric acid, fibers, and sugars, which are found at high concentration in citrus samples. For apple samples, a better correlation was found between TPC and FRAP results, in accordance with other studies.(22) 
These results were presented in a poster format that the students prepared in the last day of the traineeship. The students concluded that:

$\checkmark$ TPC and TAC depend on the matrix and the extraction conditions used;

$\checkmark$ Orange peel has a higher content of antioxidants when compared to the fruit pulp;

$\checkmark$ When peels are not edible it is important to find a process to exploit this potential source of antioxidants, and

$\checkmark$ Fruit should be consumed daily to fully take advantage of its nutritious value.

\section{Conclusions}

Evaluation of the TPC and the TAC in fruit samples introduced high school students to the following laboratory techniques: extraction, centrifugation, decantation, and spectrophotometric analysis. Students were also introduced to the preparation of standard solutions for calibration, instrument calibration, and graph interpretation and ultimately to how to determine analyte concentrations spectroscopically. In addition to the improvement of student analytical skills, an effort was made to assess the impact of this project on student perceptions and attitudes regarding scientific research. On the basis of the data collected, the students found this experience to be very positive and motivating in view of a future scientific/technological career option.

\section{Acknowledgment}

M.F.B. is grateful for the postdoctoral fellowship (SFRH/BPD/78845/2011) financed by POPHQREN-Tipologia 4.1—Formação Avançada, subsidized by Fundo Social Europeu and Ministério da Ciência, Tecnologia e Ensino Superior. The authors acknowledge the funding received through Projects UID/QUI/50006/2013, the bilateral project Luso-Serbia 2015/2016 "Agro-food waste valorization through 'green' extraction techniques: evaluation of bioactive compounds" and the Portuguese Science and Technology program "Internships for Young People in Laboratories (Ciencia Viva no Laboratório)".

\section{REFERENCES}

(1) Laguerre, M.; Decker, E. A.; Lecomte, J.; Villeneuve, P. Methods for evaluating the potency and efficacy of antioxidants. A review. Curr. Opin. Clin. Nutr. Metab. Care 2010, 13 (5), 518-525.

(2) Lobo, V.; Patil, A.; Phatak, A.; Chandra, N. Free radicals, antioxidants and functional foods: Impact on human health. Pharmacogn. Rev. 2010, 4 (8), 118-126. 
(3) Singh, R.; Devi, S.; Gollen, R. Role of free radical in atherosclerosis, diabetes and dyslipidaemia: larger-than-life. Diabetes/ Metab. Res. Rev. 2015, 31, 113-126.

(4) Turati, F.; Rossi, M.; Pelucchi, C.; Levi, F.; La Vecchia, C. Fruit and vegetables and cancer risk: a review of southern European studies. Br. J. Nutr. 2015, 113 (S2), S102-S110.

(5) Hoch, M. A.; Russell, C. B.; Steffen, D. M.; Weaver, G. C.; Burgess, J. R. Assessment of Antioxidant Capacities in Foods: A Research Experience for General Chemistry Students. J. Chem. Educ. 2009, 86 (5), 595-597.

(6) Rubio, L.; Motilva, M. J.; Romero, M. P. Recent advances in' biologically active compounds in herbs and spices: a review of the most effective antioxidant and anti-inflammatory active principles. Crit. Rev. Food Sci. Nutr. 2013, 53 (9), 943-953.

(7) Tiwari, U.; Cummins, E. Factors influencing levels of phytochemicals in selected fruit and vegetables during pre-and postharvest food processing operations. Food Res. Int. 2013, 50 (2), 497-506.

(8) Rabetafika, H. N.; Bchir, B.; Blecker, C.; Richel, A. Fractionation of apple by-products as source of new ingredients: Current situation and perspectives. Trends Food Sci. Technol. 2014, 40, 99-114.

(9) Mamma, D.; Christakopoulos, P. Biotransformation of Citrus ByProducts into Value Added Products. Waste Biomass Valorization 2014, 5 (4), 529-549.

(10) Mann, F. M. Identification and analysis of bioactive components of fruit and vegetable products. J. Chem. Educ. 2015, 92, 892-895.

(11) Galloway, K. R.; Bretz, S. L.; Novak, M. Paper chromatography and UV-Vis spectroscopy to characterize anthocyanins and investigate antioxidant properties in the organic teaching laboratory. J. Chem. Educ. 2015, 92, 183-188.

(12) Barroso, M. F.; de-los-Santos-Álvarez, N.; Delerue-Matos, C.; Oliveira, M. B. P. P. Towards a reliable technology for antioxidante capacity and oxidative damage evaluation: electrochemical (bio)- sensors. Biosens. Bioelectron. 2011, 30, 1-12.

(13) Apak, R.; Gorinstein, S.; Böm, V.; Schaich, K. M.; Özyürek, M.;Güclư“; K. Methods of measurement and evaluation of natural antioxidant capacity/activity (IUPAC Technical Report). Pure Appl.Chem. 2013, 85 (5), 957-998. 
(14) Apak, R.; Özyürek, M.; Güclu̧“, K.; Çapanoğlu, E. Antioxidant activity/capacity measurement. 1. Classification, physicochemical principles, mechanisms, and electron transfer (ET)-based assays. J. Agric. Food Chem. 2016, 64 (5), 997-1027.

(15) Oroian, M.; Escriche, I. Antioxidants: Characterization, natural sources, extraction and analysis. Food Res. Int. 2015, 74, 10-36.

(16) Ciência Viva no Laboratório. http://www.cienciaviva.pt/ocif/ (accessed Apr 2017).

(17) Alves, C. C. Learning Science Through Work Experience: Ciencia Viva Science Internships Program for Senior Secondary School Students. Int. J. Sci. Society 2012, 3 (1), 13-26.

(18) Barroso, M. F.; Ramalhosa, M. J.; Alves, R. C.; Dias, A.; Soares, C. M. D.; Oliva-Teles, M. T.; Delerue-Matos, C. Total antioxidante capacity of plant infusions: Assessment using electrochemical DNAbased biosensor and spectrophotometric methods. Food Control 2016, 68, 153-161.

(19) Shaver, L. A.; Leung, S. H.; Puderbaugh, A.; Angel, S. A. Two Methods of Determining Total Phenolic Content of Foods and Juices in a General, Organic, and Biological (GOB) Chemistry, Lab. J. Chem. Educ. 2011, 88 (4), 492-495.

(20) Rusak, G.; Komes, D. E.; Likic, S.; Horzic, D.; Kovac, M. Phenolic content and antioxidative capacity of green and white tea extracts depending on extraction conditions and the solvent used. Food Chem. 2008, 110, 852-858.

(21) Valavanidis, A.; Vlachogianni, T.; Psomas, A.; Zovoili, A.; Siatis, V. Polyphenolic profile and antioxidant activity of five apple cultivars grown under organic and conventional agricultural practices. Int. J. Food Sci. Technol. 2009, 44 (6), 1167-1175.

(22) Goulas, V.; Manganaris, G. A. Exploring the phytochemical content and the antioxidant potential of Citrus fruits grown in Cyprus. Food Chem. 2012, 131 (1), 39-47. 